\title{
LAMINAR BOUNDARY LAYER ON A CONE WITH UNIFORM INJECTION
}

Hard copy $(\mathrm{HC}) \$ 100$

Microfiche (MF)
Paul A. Libby University of California, San Diego La Jolla, California

\section{9}
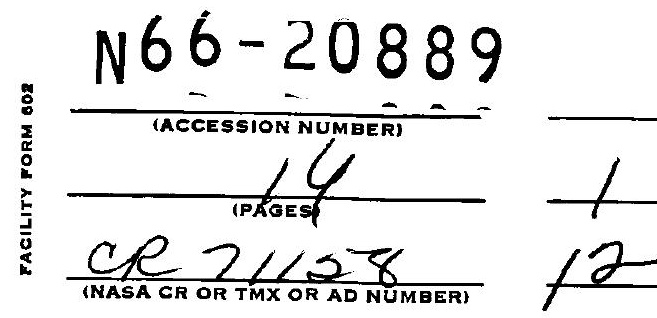

ff 653 July 65

A solution for the laminar boundary layer on a cone with uniform mass transfer is obtained. The velocity field is found for either suction or injection but the related solution for the energy field is subject to an energy balance at the exposed surface and is therefore valid only for injection. This latter solution is equally applicable to certain species fields as well. The present results along with those presented previously for the two-dimensional case permit a comparison of the effect of injection on boundary layers over twodimensional and conical surfaces.

\section{INTRODUCTION}

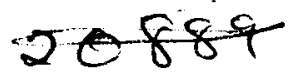

In a previous paper ${ }^{l}$ the present author and $K$. Chen provide an analysis of the laminar compressible boundary layer with uniform injection on a two-dimensional surface. The velocity field is applicable to uniform suction as well but the related solutions for the energy and species conservation, since they satisfied energy and mass balance conditions at the exposed surface, apply only to the case of injection. This is perhaps the more interesting case from an applied point of view. It is pointed out there that a similar, indeed formally identical, solution can be carried out for the flow on a cone with uniform injection, i. e., $(\mathrm{ov})_{\mathrm{w}}=$ constant. It is the purpose of this

\footnotetext{
IP. A. Libby and K. Chen, Phys. Fluids 8 , 568 (1965).
} 
note to present the numerical results of this companion study; there appear to be no previous analyses of this case. The presentation can be made rather succinctly by referring to the previous paper and by pointing out the differences in the two analyses. 2

\section{THE VELOCITY FIELD}

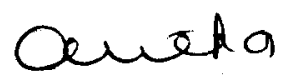

For a cone the cylindrical radius $r=x \sin \theta_{c}=\alpha x$ where $\theta_{c}$ is the half-angle of the cone and $x$ is the coordinate along the surface of the cone. The solutions are again found in terms of the transformed boundary layer variables $s, n$ but for the present case

$$
\begin{aligned}
& n \equiv o_{\mathrm{e}} \mathrm{u}_{\mathrm{e}}^{\mathrm{r}(2 \mathrm{~s})^{-1 / 2} \int_{0}^{\mathrm{y}}\left(\rho^{\prime} / o_{\mathrm{e}}\right) \mathrm{d} \mathrm{y}^{\prime}} \\
& \mathrm{s} \equiv \rho_{\mathrm{e}} \mu_{\mathrm{e}} \mathrm{u}_{\mathrm{e}} \int_{0}^{\mathrm{x}} \mathrm{r}^{2} \mathrm{dx}=o_{\mathrm{e}} \mu_{\mathrm{e}} \mathrm{u}_{\mathrm{e}} \alpha^{2}\left(\mathrm{x}^{3} / 3\right) .
\end{aligned}
$$

The solution for the velocity field is again carried out in terms of the modified stream function $F(s, \eta)$ defined by Eq. (4) of the previous paper, subject to the same initial condition, to the same condition at $\eta \rightarrow \infty$, and to the same no-slip condition at the surface $\eta=0$. However, the final boundary condition at $\eta=0$ is now altered; for the case of uniform mass transfer, i.e., for $(\rho v)_{\mathrm{w}}=$ constant, the equation relating the $v$-velocity component to $F(s, \eta)$ leads in the case of the cone to

$F(s, 0)=\left\{\left[-(\rho v)_{w} / \rho_{e} u_{e}\right]\left(o_{e} u_{e}\right)^{1 / 3} \mu_{e}-2 / 3\left(3^{2 / 3} / \alpha^{1 / 3} 2^{3 / 2}\right)\right\} s^{1 / 6}$

compared to $F(s, 0) \propto s^{1 / 2}$ for the two-dimensional case. As in the previous analysis, it is convenient to introduce a new nondimensional, independent variable, which is denoted here by a separate symbol $X$ so as to distinguish it from the previous, independent variable, $\epsilon(s)$; let

$$
x=\{\} s^{1 / 6}
$$

2 The notation of the previous paper is carried over without change; only new quantities are, therefore, defined here. 
where $x>0$ for suction and $x<0$ for injection and where the \{\} factor is defined by the right-hand side of Eq. (2). Note that if the mass transfer rate $(\rho v)_{\mathrm{w}} / \rho_{\mathrm{e}} \mathrm{u}_{\mathrm{e}}$ is specified, if the properties of the external stream in terms of $o_{\mathrm{e}}, \mu_{\mathrm{e}}, \mathrm{u}_{\mathrm{e}}$ are known, and if the geometry of the cone in terms of $\alpha$ is known, then $X=X(x)$ so that the solutions in terms of $X$ can be directly related to the station along the cone defined by $x$.

Now consider $F$ to be a function of $X$ and $\eta$. The final equation and conditions are then

$$
\mathrm{F}_{n \eta \eta}+\mathrm{FF}_{\eta \eta}-(\mathrm{X} / 3)\left(\mathrm{F}_{\eta} \mathrm{F}_{\eta \mathrm{X}}-\mathrm{F}_{\mathrm{X}} \mathrm{F}_{\eta \eta}\right)=0
$$

subject to the conditions

$$
\begin{aligned}
& F_{\eta}(0, \eta)=F_{0}^{\prime}(\eta), \quad F_{\eta}(X, 0)=0, \quad F_{n}(X, \infty)=1 \\
& F(X, 0)=X .
\end{aligned}
$$

The skin-friction coefficient, a quantity of applied interest, is now related to $F$ and to $X$ by the equation

$$
c_{f}=(3 / 2)\left[(-o v)_{w} / 0 e^{u} e^{X]\left(F_{\eta \eta}\right)_{w}} .\right.
$$

As in the previous analysis the solution to Eq. (4) is found in the form

$$
F(x, \eta)=F_{0}(\eta)+\sum_{n=1}^{\infty} x^{n} N_{n}(\eta)
$$

Substitution of Eq. (6) into Eq. (4) leads as previously to an array of equations of the form

$$
\begin{aligned}
N_{n}^{\prime \prime \prime}+F_{0} N_{n}^{\prime \prime}-(n / 3) F_{0}^{\prime} N_{n}^{\prime}+[1+(n / 3)] F_{0}^{\prime \prime} N_{n} & =0, n=1 \\
& =R_{n}^{(1)}, \quad n \geq 2
\end{aligned}
$$


which is subject to the conditions

$$
\begin{aligned}
N_{n}^{\prime}(0) & =N_{n}^{\prime}(\infty)=0 \\
N_{n}(0) & =1, \quad n=1 \\
& =0, \quad n \geq 2 .
\end{aligned}
$$

The right-hand sides, i.e., the $R_{n}^{(1)}$, for $n \geq 2$ depend on the previous functions in the array.

The first five functions $N_{n}(n)$ have been found numerically. The crucial values for generating these functions in detail are $\mathrm{N}_{n}^{\prime \prime}(0)$; ac cordingly, these have been listed in Table I. In addition the "velocity profiles" are usually of most graphic interest so these have been given in Fig. 1. Also of interest is the variation of $\left(F_{n \eta}\right)_{w}$ with $x$; this is shown in Fig. 2 for four and five terms in the series. As in the previous case the value of $x<0$ for which $\left(F_{\eta \eta}\right)_{w}=0$, i.e., the "blow-off" value, although of conceptual and perhaps of applied interest, cannot be determined by the present analysis. This completes consideration of the velocity field.

Table I. Initial values of $N_{n}(n)$ functions.

\begin{tabular}{cc}
\hline $\mathrm{n}$ & $\mathrm{N}_{\mathrm{n}}^{\prime \prime}(0)$ \\
\hline 1 & 0.9039 \\
2 & 0.2504 \\
3 & -0.1068 \\
4 & 0.04038 \\
5 & -0.07963 \\
\hline
\end{tabular}

III. THE ENERGY FIELD

Under the same assumptions relative to transport properties of the gas used in the previous paper, the equation of energy conservation 
in terms of the ratio of stagnation enthalpies $g \equiv h_{s} / h_{s, e}$ and of $X$ and $\eta$ is

$$
g_{\eta \eta}+F_{\eta}-(x / 3)\left(F_{\eta} g_{X}-F_{X} g_{\eta}\right)=0
$$

As previously it will be assumed that only the case of injection $(x<0)$ is of interest and that the convective heat transfer from the gas to the exposed surface is absorbed by the coolant in passing from the coolant chamber where its enthalpy ratio is $g_{c}$ to the exposed surface where its enthalpy ratio is $\mathrm{g}_{\mathrm{w}}=\mathrm{g}_{\mathrm{w}}(X)$, to be determined. On physical grounds it is anticipated that $\mathrm{g}_{\mathrm{W}}(0)=\mathrm{l}$ so that the initial and boundary conditions are

$$
\begin{aligned}
& g(x, \infty)=g(0, \eta)=1 \\
& (4 / 3)(-x)\left(g_{w}-g_{c}\right)=\left(g_{\eta}\right)_{w} .
\end{aligned}
$$

The boundary condition at $\eta=0$ represents the heat balance condition for the present case of a cone. It is perhaps worth noting that if in addition to the convective heat load there exists a uniform additional thermal load, e.g., due to radiation, then this heat balance condition still prevails but with a redefincd $g_{c}$ which accounts for this added load.

The solution of Eq. (8) subject to the conditions of Eqs. (9) is found in the form

$$
g(x, \eta)=1+\left(g_{c}-1\right) \sum_{n=1}^{\infty} x^{n} G_{n}(\eta)
$$

where the $G_{n}(\eta)$ functions are given by an array of ordinary differential equations

$$
\begin{aligned}
G_{n}^{\prime \prime}+F_{0} G_{n}^{\prime}-(n / 3) F_{0}^{\prime} G_{n} & =0, \quad n=1 \\
& =R_{n}^{(2)}, \quad n \geq 2
\end{aligned}
$$

and are subject to the conditions 


$$
\begin{aligned}
G_{n}^{\prime}(0) & =-(4 / 3) G_{n-1}(0), & & n \geq 2 \\
& =(4 / 3), & & n=1 .
\end{aligned}
$$

Again the $R_{n}^{(2)}$ functions are known functions of the previous $N_{n}$ and $G_{n}$ functions.

The first five $G_{n}$ solutions have been obtained numerically; for their reproduction by straightforward numerical integration the values of $G_{n}(0)$ and $G_{n}^{\prime}(0)$ are listed in Table II. For graphic displace the dis tributions of $G_{n}(\eta)$ are shown in Fig. 3 while the distribution of wall enthalpy in the form $\left(1-g_{w}\right)\left(1-g_{c}\right)^{-1}$ as given by four and five terms in the series is shown in Fig. 4.

Table II. Initial values of $G_{n}(\eta)$ functions.

\begin{tabular}{cll}
\hline $\mathrm{n}$ & $\mathrm{G}_{\mathrm{n}}^{\prime}(0)$ & $\mathrm{G}_{\mathrm{n}}(0)$ \\
\hline 1 & 1.333 & -2.465 \\
2 & 3.2 .87 & -1.645 \\
3 & 2.193 & -0.2046 \\
4 & 0.2728 & -0.1205 \\
5 & 0.1607 & -0.1214 \\
\hline
\end{tabular}

IV. CONCLUDING REMARKS

In conclusion perhaps several remarks will be pertinent. As in the previous paper the concentration field, in terms of species mass fractions in cases with no gas-phase reaction and of element mass fractions in cases with such reaction, is given by the solution for the energy field provided, as is physically reasonable, a mass balance at the exposed surface is imposed. 
It is perhaps of interest in applications to compare $\epsilon(s)$ and $x(s)$; for this purpose consider a wedge and a cone under flow conditions so that $\rho_{\mathrm{e}} \mu_{\mathrm{e}}, \mathrm{u}_{\mathrm{e}}$ are the same in the two cases and let $(0 \mathrm{v})_{\mathrm{w}} / o_{\mathrm{e}} \mathrm{u}_{\mathrm{e}}$ be common. Then at equal distances from the leading edge and from the apex, i.e., at the same station $x$, it can be shown that

$$
\epsilon\left(s_{1}\right)=3^{1 / 2} \times\left(s_{2}\right)
$$

where $s_{1}$ and $s_{2}$ are the values of $s$ for the wedge and cone, respectively. In addition, the total mass added through the porous surface from the apex of the cone to the generic station $x$ per unit length of perimeter at $x$ is one-half that added from the leading edge of the cone over the same $x$-wise length per unit length parallel to the leading edge. If Figs. 2 and 4 are compared, respectively, with Figs. 5 and 9 of the previous paper in terms of these considerations of the wedge and cone, it is found that the skin-friction on the cone is altered less by mass transfer and the wall enthalpy on the cone with injection is higher than on the wedge.

\section{ACKNOW LEDGMENTS}

The author gratefully acknowledges that Mrs. Judith Hays of the UCSD Computer Center carried out the numerical analysis. Ine resuits in this note have been obtained as part of a study being carried out under NASA Grant NGR 05-009-025. 
List of Figures

1. The function $\mathrm{N}_{\mathrm{n}}^{\prime}(\eta)$

2. The effect of mass transfer on the skin-friction parameter

3. The function $G_{n}(\eta)$

4. The variation of wall enthalpy with the injection parameter 


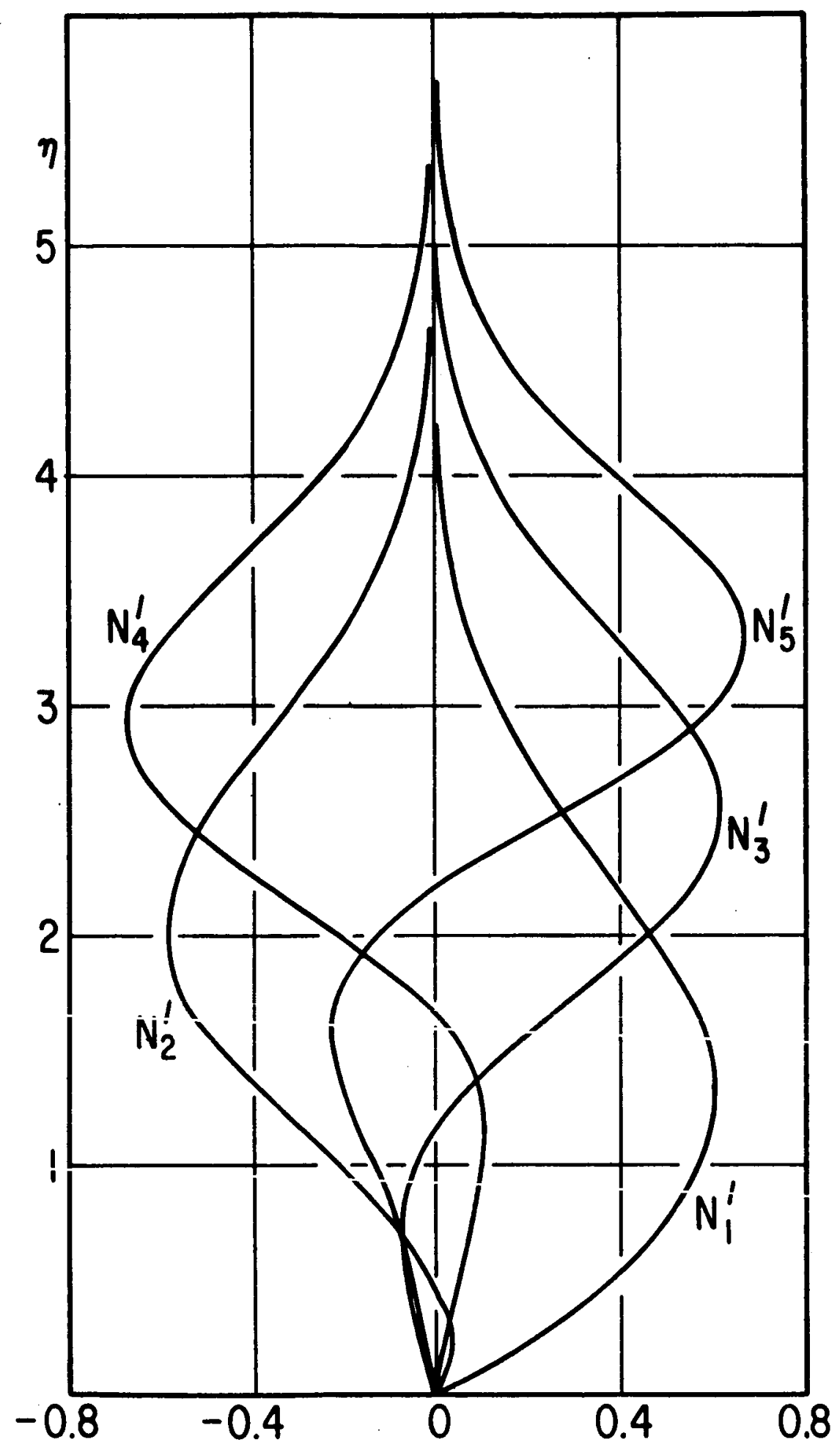




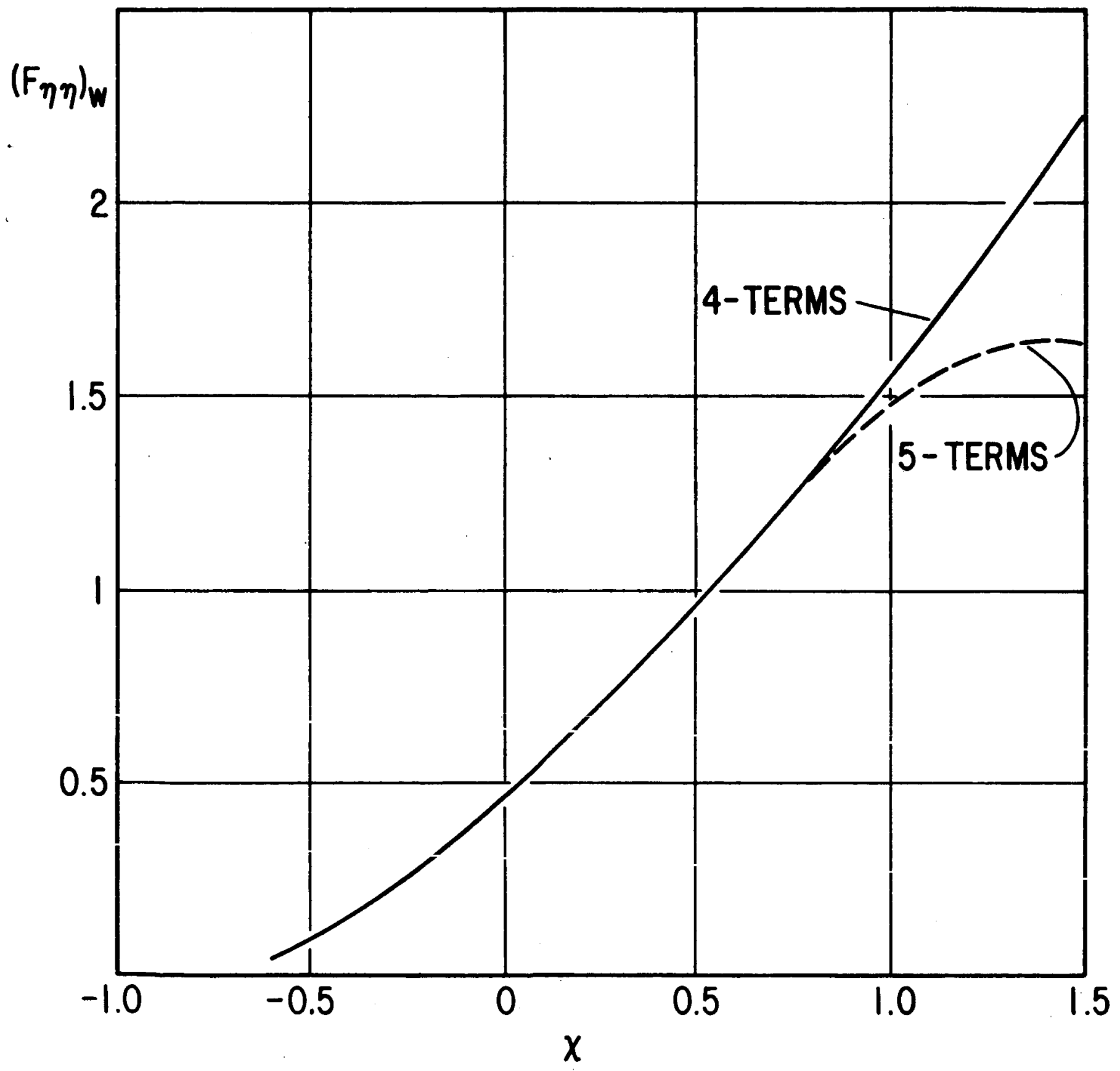




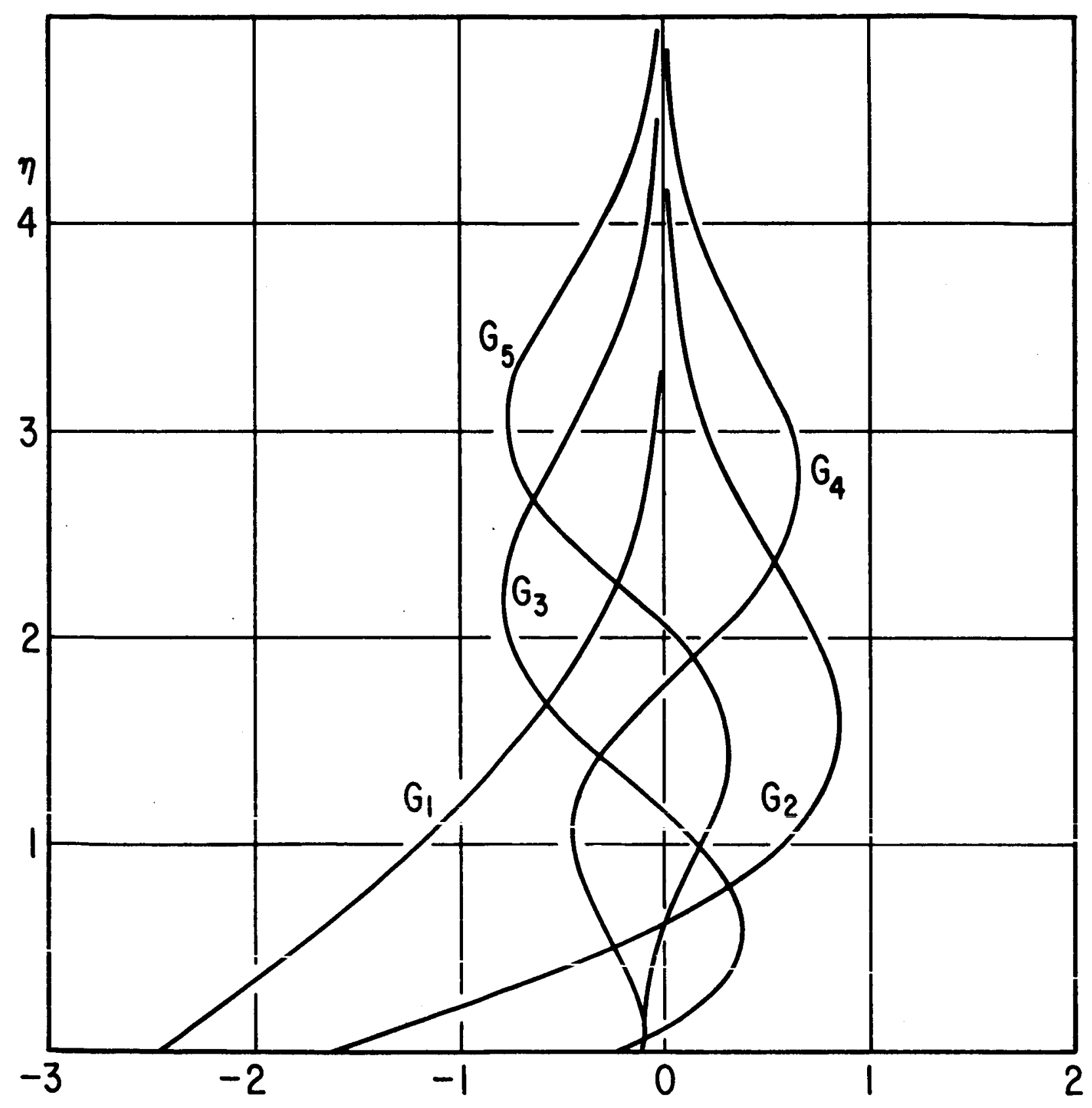




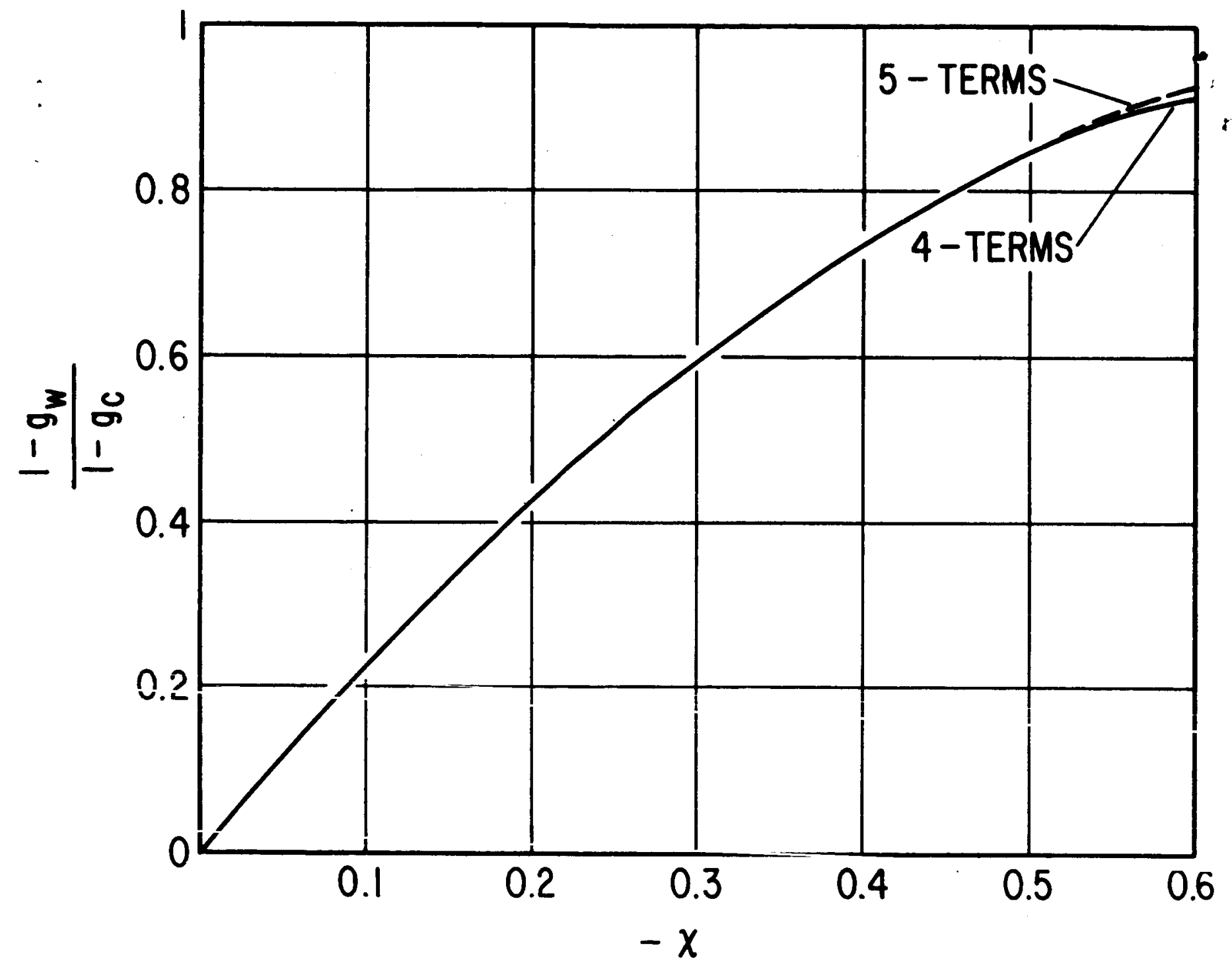

